\title{
Resultados clínicos de la resincronización ventricular en pacientes con insuficiencia cardíaca severa
}

\author{
René Asenjo1,2, Mario Zapata1, Ivonne Aramburú1, \\ Raimundo Morris ${ }^{1}$, Mauricio Cereceda ${ }^{1}$, Solange Brugere ${ }^{1}$, \\ Rubén Aguayo ${ }^{1}$, Jhonny Ríos $^{1}$, Carlos Toro ${ }^{1}$, Mario Hassi ${ }^{3}$, \\ Patricio G onzález ${ }^{1}$, José Cannessa ${ }^{3}$, Rodulfo 0 yarzún ${ }^{1,2}$, \\ Mario 0 rtiz $^{1}$, Viviana Avalos ${ }^{\mathrm{a}}$, Patricia Morales ${ }^{\mathrm{a}}$, \\ Martín Nicolab ${ }^{b}$ Miguel 0 yonarte ${ }^{1}$ y Alejandro Abufhele ${ }^{2}$. \\ Results of intraventricular \\ resynchronization in patients with severe \\ cardiac failure
}

Background: Intraventricular resynchronization with pacemakers is a promising therapy for patients with refractory cardiac failure and intraventricular conductions delay. However its long term effects are not well known. Aim: To report the results of this therapy in patients with cardiac failure. Patients and methods: Fourteen patients (11 male), whose mean age was 68 years, with a severe and refractory cardiac failure, have been treated in our unit using intraventricular resynchronization with pacemakers. Eight had a coronary heart disease and six a dilated myocardiopathy. The pacemaker was implanted transvenously, with conventional stimulation in atrium and right ventricle. The left ventricle was stimulated through an epicardial vein, accessed through the coronary sinus. Results: In one patient the high thresholds did not allow a left ventricular stimulation. In the other 13 patients, a clinical improvement was observed in 11 (85\%), that has been sustained for a mean of 8.2 months. The ejection fraction improved form 23.5 to $32.4 \%$ ( $\mathrm{p}<0.001$ ), the 6 min walking test improved from 347 to $437 \mathrm{~m}(\mathrm{p}=0.003)$ and the functional capacity changes from 3.3 to 2.7 ( $\mathrm{p}$ $\varangle 0.001$ ). Three patients died during follow up. One was the patient in whom the stimulation failed and two had a sudden death. No complications of the procedure were observed. Conclusions: In this series, intraventricular resynchronization with pacemakers was effective in 11 of 13 patients, improving functional capacity and ejection fraction. Sudden death could be avoided adding a defibrillator to the pacemaker system (Rev Méd Chile 2003; 131: 1101-10).

(Key Words: Cardiac surgical procedures; Cardiac pacing, artificial; Pacemaker, artificial).

\footnotetext{
Recibido el 1 de abril, 2003. Aceptado en versión corregida el 4 de agosto, 2003.

${ }^{1}$ Unidad de Arritmias, Centro Cardiovascular, Hospital Clínico, Facultad de Medicina, Universidad de Chile,

${ }^{2}$ Laboratorio de Electrofisiología, Clínica Alemana,

${ }^{3}$ Departamento de Cardiología, Hospital Dipreca, Santiago de Chile.

aEnfermera Universitaria, Unidad de Arritmias, Hospital Clínico Universidad de Chile.

bAlumno de la Escuela de Medicina, Sede Norte, Universidad de Chile.
}

\footnotetext{
Correspondencia a: Dr. René Asenjo G. Dirección: Santos

Dumontt N999, Independencia, Santiago - Chile. Fonos:

7378038-6788357. Fax: 7320683.

E mail: cenaar@ns.hospital.uchile.cl / reneasenjo@vtr.net
} 
A pesar de los grandes avances terapéuticos, el desarrollo de insuficiencia cardíaca (IC) es una complicación inevitable en una importante proporción de pacientes, y su alta prevalencia la ha transformado en un problema creciente de salud mundial ${ }^{1}$. No se conocen cifras reales de IC en Chile, pero en los Estados Unidos de Norteamérica se presentan 500.000 casos nuevos cada año, y más de 250.000 pacientes mueren anualmente por esta causa, principalmente por muerte súbita (MS) amítmica y refractariedad de la IC 2 . El desarmollo del desfibrilador interno (DI) ha significado un gran aporte para disminuir la $\mathrm{MS}^{3,4}$, pero no modifica la evolución de la IC. El trasplante cardíaco surge entonces como la única terapia capaz de cambiar el pronóstico de la enfermedad, mejorando notablemente la sobrevida, capacidad funcional (CF) y calidad de vida, no obstante la cantidad de candidatos sobrepasa por mucho la disponibilidad de donantes ${ }^{5}$.

Una importante proporción de enfermos con disfunción sistólica de ventrículo izquierdo (VI), tienen retardo de la conducción intraventricular (CIV), el que se manifiesta por ensanchamiento del QRS, y que ha sido reconocido como un marcador independiente de mal pronóstico en pacientes con IC $^{6}$. Este retardo de la CIV puede llevar a que segmentos del VI y ventrículo derecho (VD) se contraigan en tiempos distintos de lo que normalmente ocurre, lo que se define como disincronía ventricular ${ }^{6}$, y que produce disminución de la contractilidad del VI, tiempo de llenado diastólico reducido, y aumento del reflujo mitral, causando mayor deterioro hemodinámico y funcional en un corazón ya comprometido. La estimulación con marcapaso (MP) en múltiples sitios, o biventricular (BiV), denominada también terapia de resincronización ventricular (TRV), se ha demostrado que corrige la disincronía de la contracción ventricular ${ }^{7}$ y varios estudios han mostrado mejoría hemodinámica, de la fracción de eyección (FE), CF y calidad de vida en estos pacientes ${ }^{8-13}$; no obstante, todavía poco se conoce sobre la evolución alejada. La primera experiencia nacional en esta alentadora forma de terapia recientemente incorporada para pacientes con IC avanzada, fue presentada por nuestro grupo en el último Congreso de nuestra Sociedad $^{14}$. A la fecha, hemos aumentado el número de pacientes y el tiempo de seguimiento. Por lo tanto, el objetivo de este trabajo es analizar los resultados inmediatos y efectos alejados de la TRV en los enfermos tratados por nuestro grupo.

\section{MateRIAl y MÉTOdO}

Pacientes. Desde agosto de 1999 a agosto de 2001, catorce pacientes (11 hombres), edad promedio 67,6 años (rango 54 a 87 años), han sido seleccionados para TRV. Los criterios de inclusión para esta forma de terapia fueron: severa dilatación y disfunción de VI con $\mathrm{FE} \leq 30 \%$, trastorno de CIV con QRS >150 ms o portador de MP ventricular previo, y severa limitación de la CF con IC CF III o IV a pesar de terapia máxima optimizada, que incluyó inhibidores de la enzima convertidora, espironolactona y betabloqueadores, excepto contraindicación para su uso ${ }^{5,6}$. En todos se realizó estudio ecocardiográfico completo y determinación clínica de $\mathrm{CF}$, y en los que fue posible se realizó ventriculografía isotópica y test de caminata de 6 min (este último test no se realizó en los enfermos en CF IV). Ocho tenían cardiopatía coronaria con secuelas de infarto, 6 de los cuales habían sido revascularizados, y 6 tenían miocardiopatía dilatada (Tabla 1). Diez estaban en CF III

\section{Tabla 1. Resincronización ventricular en pacientes con IC severa}

\begin{tabular}{|lc|}
\hline Características generales: & \\
\hline Número de pacientes & 14 \\
Hombres & 11 \\
Mujeres & 3 \\
Edad rango: 54 a 87años & 67,6 \\
Cardiopatía de base & \\
$\quad$ Coronaria & 8 \\
$\quad$ Miocardiopatía dilatada & 6 \\
Capacidad funcional al implante & 10 \\
$\quad$ III & 4 \\
IV & 9 \\
Trastorno de conducción & 1 \\
$\quad$ Bloqueo completo rama izquierda & \\
$\quad$ Trastorno de conducción inespecífico & 1 \\
$\quad$ Marcapaso previo & \\
Ritmo cardíaco de base & 13 \\
$\quad$ Sinusal & 1 \\
$\quad$ Fibrilación auricular & 3 \\
Antecedente de taquicardia ventricular & \\
\hline
\end{tabular}


y 4 en CF IV, estos últimos con apoyo inotrópico intravenoso prolongado y uno, adicionalmente, en ventilación mecánica (VM) por la IC. El trastorno de CIV fue bloqueo completo de rama izquierda (BCRI) en 9 pacientes, trastorno inespecífico de la CIV en 1 y 4 tenían un MP previo, 3 DDD y uno VVI; este último paciente estaba en fibrilación auricular (FA). Tres tenían antecedentes de taquicardia ventricular (TV) sostenida con severo compromiso hemodinámico.

Implante del sistema de TRV. En todos los pacientes el implante se hizo por vía transvenosa; en aquellos que no tenían MP previo se implantó un electrodo en aurícula y ventrículo derecho (VD) en forma convencional, ubicando éste último preferentemente en el septum alto $^{5}$. El electrodo para estimulación de VI se ubicó en una vena epicárdica a la que se accedió vía seno coronario (SC) (Figura 1). Se seleccionó preferentemente una vena lateral o póstero lateral ${ }^{8-13}$ en la que hubiera estabilidad del electrodo, y además un umbral de estimulación $\leq$ 2,5 V y ausencia de estimulación diafragmática (Figura 2). En los 4 pacientes que tenían MP previo la estimulación BiV se logró con el uso de un conector en $\varangle \gg$ (Medtronic, MN, USA) a través del cual se conectaron el terminal del electrodo de VD y de VI al canal ventricular del MP para estimulación BiV simultánea. En los enfermos restantes se implantó un sistema tricameral especialmente diseñado para este tipo de estimulación: Insync, Medtronic, MN, USA en 5 y Contak, Guidant, MN, USA, en los otros 4. En los 3 pacientes con TV el MP incluyó un DI incorporado en el sistema (Contak CD, Guidant).

En 2 pacientes muy graves e inestables la intervención se realizó bajo anestesia general; en los otros se usó anestesia local y sedación intravenosa.

Programación del Sistema de TRV y Seguimiento. Luego del implante, el MP se programó para asegurar estimulación BiV permanente ${ }^{5}$. En los pacientes en ritmo sinusal el MP se programó con frecuencia mínima que permita un funcionamiento predominante como VDD. El intervalo aurículoventricular (IAV) se programó con guía de Eco-Doppler buscando la mejor curva de llenado ventricular y la mejor curva de eyección aórti$\mathrm{ca}^{5,15}$. En el paciente con FA se realizó ablación

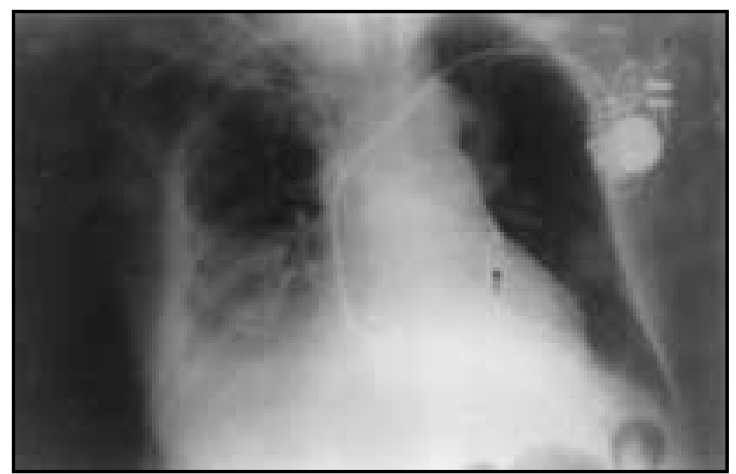

FIgURA 1. Rx tórax anteroposterior (AP) del primer paciente tratado con TRV. El MP es DDD convencional y se usó un conector en $\varangle \gg$ para unir el electrodo de VI y VD. El electrodo de seno coronario ( $\uparrow$ ) está en una rama anterior.

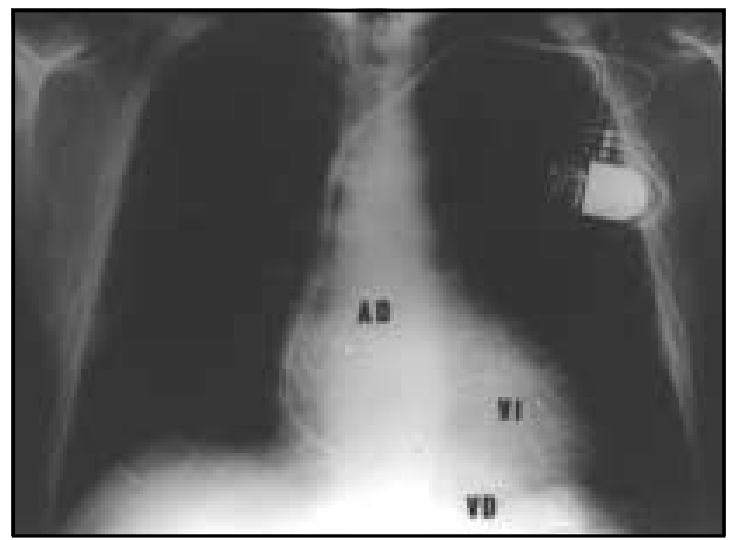

FiguRA 2. Radiografía de tórax AP con un MP especialmente diseñado para TRV. AD corresponde al electrodo en aurícula derecha, VD al electrodo de ventrículo derecho y VI muestra el electrodo en una rama póstero lateral de ventrículo izquierdo al que se accedió vía seno coronario.

del nódulo AV con radiofrecuencia para permitir estimulación ventricular exclusiva del MP, pues tenía períodos con conducción AV normal ${ }^{16}$.

La terapia farmacológica previa se mantuvo sin cambios y luego del alta los pacientes han sido controlados periódicamente en la Clínica de MP de nuestro centro. Además del control clínico y del MP, a los 2 ó 3 meses se controló el EcoDoppler y el test de caminata de 6 min y, en los que fue posible, se hizo estudio de medicina 
nuclear con ventriculografía de equilibrio gatillada comparando FE basal y luego de $5 \mathrm{~min}$ de estimulación BiV para determinar los efectos inmediatos de la TRV7

Estadística. Los resultados de la evaluación clínica y de los exámenes de control fueron comparados con los parámetros basales usando la prueba t de Student. Un valor de $\mathrm{p}<0,05$ fue considerado significativo.

\section{RESULTADOS}

Resultados inmediatos. De los 14 pacientes en que se intentó la TRV, en 13 se obtuvieron los parámetros previamente definidos para instalar el sistema. En un paciente con miocardiopatía dilatada y MP DDD previo no se encontraron umbrales adecuados en ninguna de las venas exploradas por lo que no pudo ser sometido a TRV. Una paciente requirió una segunda intervención pues en el primer intento no se logró avanzar el electrodo en el SC con los materiales disponibles en ese momento. En 2 pacientes no se logró estimulación de VI en una de las venas laterales o póstero laterales, dejando el electrodo en una rama anterior. Con la estimulación BiV la duración del QRS disminuyó de 206,2 \pm 31 ,2 a 153,1 $\pm 22,8$ ms $(p<0,05)$ (Figura 3). En todos los pacientes se observó una mejoría clínica y subjetiva dentro de las $72 \mathrm{~h}$ de la TRV. De los 3 pacientes resincronizados con apoyo inotrópico intravenoso prolongado, uno (el primer paciente tratado con esta terapia en nuestro grupo), llevaba un mes en ventilación mecánica (VM) luego de ser revascularizado quirúrgicamente. Este paciente tenía BCRI, intervalo PR largo, y severa disfunción sistólica de VI. En él se realizaron mediciones hemodinámicas en pabellón (lo que no se hizo en los demás pacientes), para definir la utilidad del sistema ${ }^{8}$, observando una disminución de la presión capilar pulmonar de 30 a $15 \mathrm{mmHg}$ y aumento de la presión sistólica de 90 a $110 \mathrm{mmHg}$, con estimulación BiV. Este paciente logró ser extubado dentro de las $48 \mathrm{~h}$ y se pudo retirar el apoyo inotrópico dentro de los 5 días siguientes, siendo dado de alta en CF III. Los otros pacientes en CF IV y con apoyo inotrópico intravenoso prolongado también lograron recuperarse rápidamente, lo que permitió retirar los fármacos inotrópicos y darles de alta antes de una semana.

El IAV «óptimo»medido por Eco-Doppler fluctuó entre 80 y $150 \mathrm{~ms}$, y en los 7 pacientes en que se realizó ventriculografía isotópica pre y post estimulación BiV se observó un aumento agudo de la FE de VI de $77,4 \%$ (22,6 \pm 10 a $40,1 \pm 9 \%)$ con TRV.

La única complicación relacionada con el implante fue un cuadro febril atribuido a hipertermia por anestesia, observado en uno de los 2 enfermos en que por la gravedad de la IC la intervención se realizó con anestesia general. No hubo desplazamiento de electrodos ni otras complicaciones.

Evolución alejada. De los 13 pacientes con TRV 11 (85\%) han tenido buena respuesta y han referido una mejoría notable, la que se ha mantenido en un seguimiento promedio de 8,2 meses (2 a 19), diez

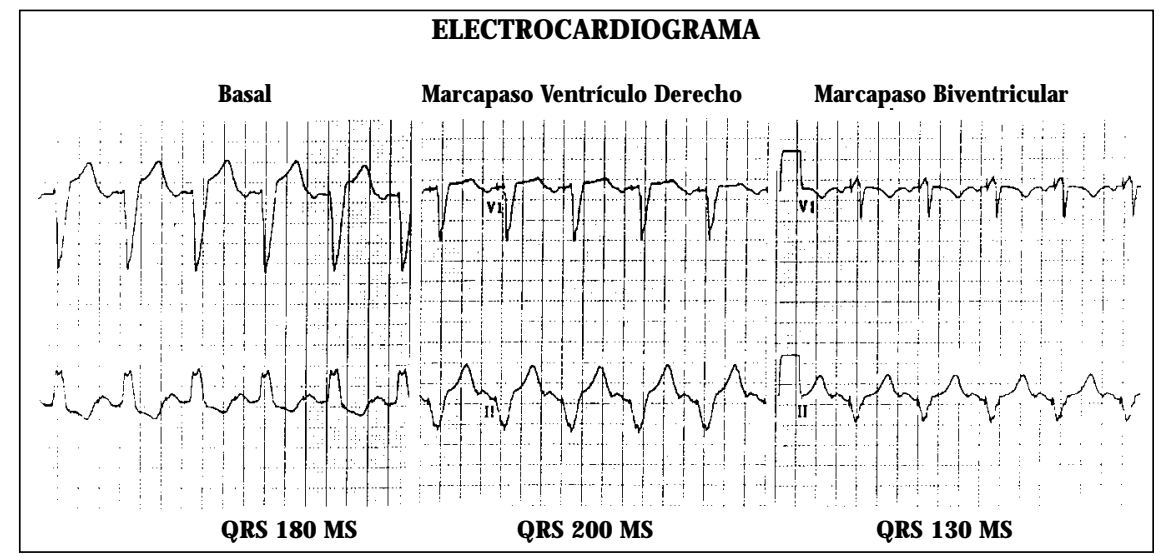

FiguRa 3. Cambios electrocardiográficos con la terapia de resincronización ventricular. Se observa los cambios morfológicos y la disminución de la duración del QRS con MP biventricular. 
en CF II y uno en CF I. En los 2 pacientes en que no se observó mejoría el electrodo para la TRV quedó en posición anterior, lo que puede explicar la mala respuesta8,11. No obstante, ambos son coronarios operados, con enfermedad coronaria de pequeño vaso residual no revascularizable, lo que también debe haber influido en su evolución.

Tres pacientes han fallecido: el paciente en que no se pudo hacer TRV murió a los 6 meses por IC refractaria, y los otros 2 fallecieron en forma súbita a los 2 y 12 meses respectivamente, luego de haber tenido buena respuesta clínica (la CF había mejorado IV a III en uno y de III a II, en el segundo). Ninguno de ellos tenía DI incorporado en su MP pues no habían tenido arritmias malignas. De los pacientes sobrevivientes, 3 están en espera de trasplante.

En la Figura 4 se muestran los cambios ecográficos de la FE y diámetro diastólico de VI. La FE

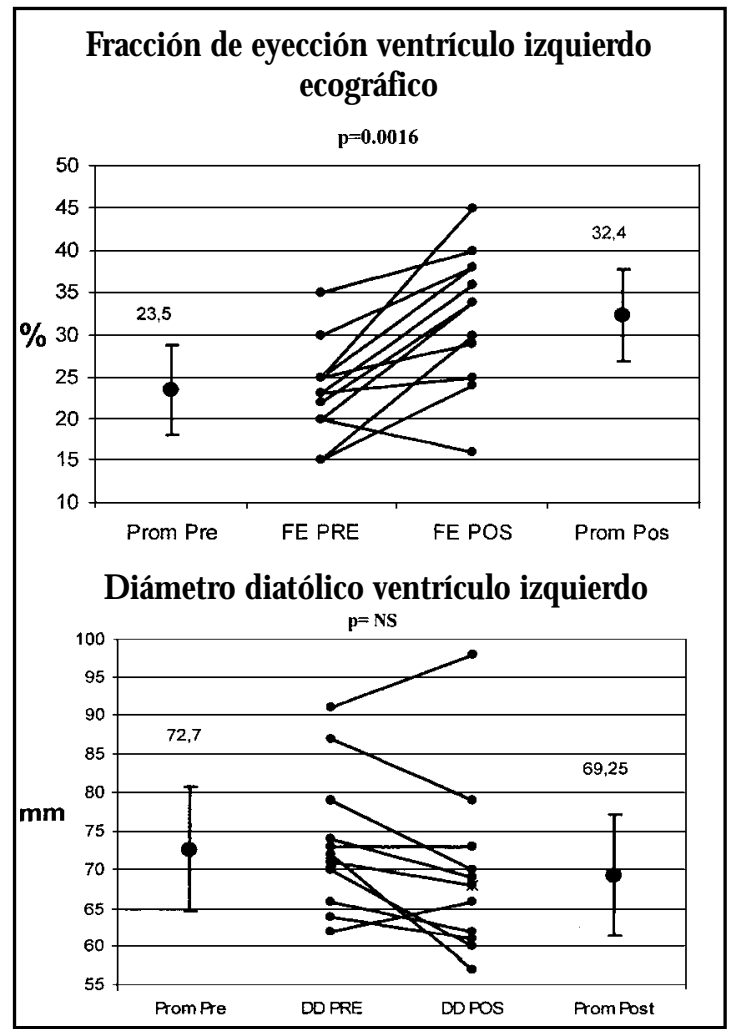

Figura 4. Se observan los cambios en la fracción de eyección y en los diámetros diastólico de VI, medidos eocográficamente, en condiciones basales y post estimulación biventricular. mejoró de 23,5 $\pm 5,4$ a 32,4+8\%, (p: 0,0016), y aunque el diámetro diastólico de VI disminuyó desde $72,7 \pm 8,6$ a $69,3 \pm 10,9 \mathrm{~mm}$, éste no alcanzó a ser estadísticamente significativo. La Figura 5 muestra los cambios en la capacidad física. El test de

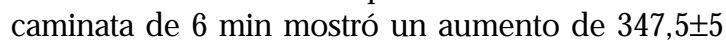
a $437,6 \pm 51 \mathrm{~m}$ y la $\mathrm{CF}$ promedio de $3,33 \pm 0,48$

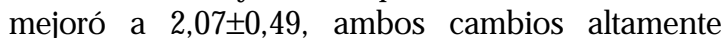
significativos (p 0,003 y 0,0015, respectivamente).

Un paciente presentó FA persistente 2 semanas post TRV y está en plan para cardioversión. En este enfermo la CF mejoró de III a II y el test de caminata de 6 min mostró aumento de 340 a 450 $\mathrm{m}$, a pesar de la arritmia.

En suma, 85\% de los 13 pacientes con TRV han experimentado mejoría post estimulación BiV, tanto subjetiva como de los parámetros ecográfi$\cos$ y funcionales controlados, la que ha sido persistente en el tiempo. No se han observado complicaciones de la TRV.

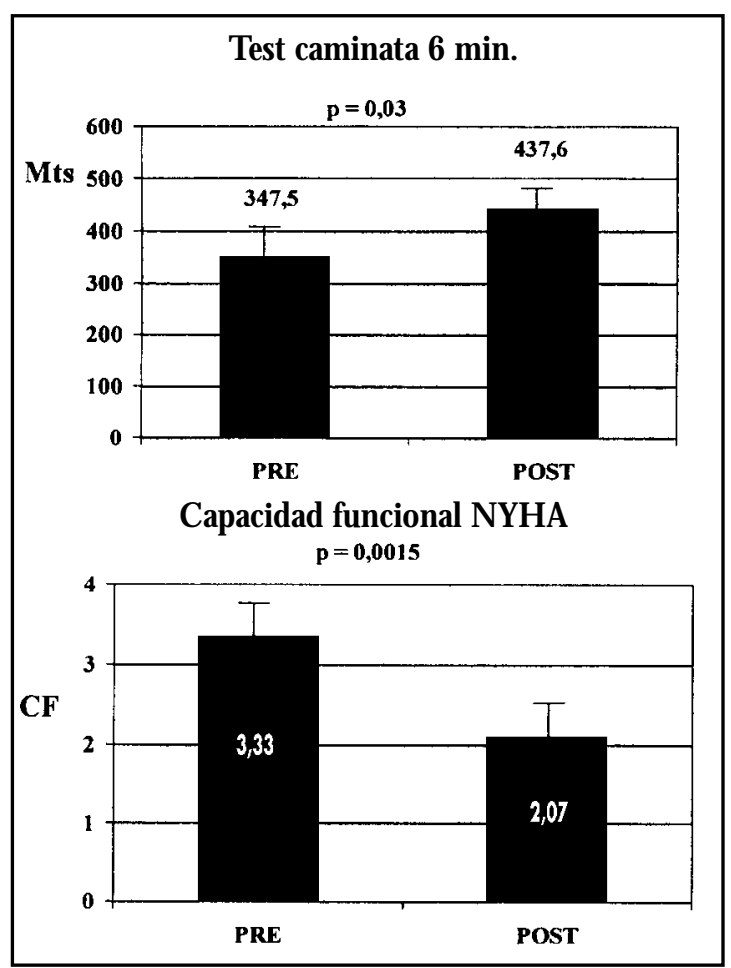

FIgURA 5. Se observan los cambios en la capacidad funcional y en el Test de Caminata de 6 min en condiciones basales y post resincronización ventricular. 


\section{DiscUSIÓN}

La presente experiencia clínica muestra que en pacientes con IC severa refractaria y trastorno de CIV la TRV produjo una mejoría notable y sostenida en la gran mayoría de los enfermos tratados. Distintos parámetros, como la FE, test de caminata de 6 min, así como la CF, muestran que esta forma de terapia recientemente introducida parece ser una alternativa válida y segura para este grupo seleccionado de pacientes gravemente enfermos y con muy alto riesgo vital en el corto plazo. Esta primera experiencia nacional es concordante con reportes provenientes de centros con mayor casuística ${ }^{10-13}$; no obstante, varios estudios multicéntricos están actualmente en marcha para definir mejor los efectos de la TRV en el más largo plazo y evaluar su rol potencial para mejorar la sobrevida.

Fundamento de la TRV. La importancia de una contracción ventricular sincronizada ha sido reconocida desde hace varias décadas. Herman y cols $^{17}$, en 1967, destacaron que más de $70 \%$ de los pacientes con asinergia tenían IC clínica. Luego, en 1983, Bramlet y cols ${ }^{18}$, determinaron mediante ventriculografía de ejercicio, que la presencia de BCRI afecta la función ventricular, aun en sujetos sin cardiopatía demostrable. En 1989 Grines y cols ${ }^{19}$ postularon que el BCRI, al alterar la activación ventricular, produce contracción retardada del VI, movimiento septal anormal, FE disminuida y llenado diastólico reducido. No obstante, fue en 1952 cuando Xiao et $\mathrm{al}^{20}$ determinaron que en pacientes con miocardiopatía dilatada un QRS prolongado se correlaciona con mayor disminución de la función sistólica y deterioro de la relajación ventricular, y postularon que si el QRS era acortado con estimulación ventricular podía mejorar la contractilidad. El impacto que estas alteraciones de la activación ventricular tienen sobre la función contráctil en enfermos con severa disfunción de VI, así como su mejoría mediante estimulación $\mathrm{BiV}$, fue sólo recientemente demostrado ${ }^{7,21}$. La TRV se asocia a mejoría de la sincronía de la contracción septal y de la pared lateral de VI, principalmente por corrección del retardo mecánico de la pared lateral ${ }^{22}$, y el grado de mejoría que se logra estaría asociado al grado de asincronía eléctrica basal ${ }^{23}$. La liberación de la mayor tensión parietal regional producida por dicho retardo de activación, lleva a una contracción ventricular más efectiva, con disminución de la demanda de $\mathrm{O}_{2}$ miocárdico, todo lo cual explicaría el «emodelamiento ventricular reverso», recientemente descrito en estos pacientes ${ }^{24}$. Así, a los efectos agudos de la TRV se sumarían disminución de los diámetros y volúmenes de VI y de los índices de función ventricular en el largo pla$\mathrm{zo}^{24,25}$, cuya repercusión sobre el pronóstico de estos pacientes está por definirse.

Experiencias clínicas con la TRV. La primera experiencia clínica con TRV fue comunicada por Cazeau y $\operatorname{cols}^{8}$ en 1996 en una serie de 8 pacientes en CF IV, obteniendo mejoría hemodinámica y clínica. Leclercq y $\operatorname{cols}^{9}$ encontraron resultados agudos similares en 18 pacientes con IC severa y trastorno de CIV. Los hallazgos de estos autores son similares al efecto hemodinámico agudo que nosotros observamos en el primer enfermo que tratamos con TRV en 1999. Grass y cols ${ }^{10}$ comunicaron una experiencia no randomizada de 81 pacientes en 1998, reportando beneficio clínico con estimulación BiV en pacientes en CF III o IV, el que se mantuvo hasta los 12 meses de seguimiento. Luego Auricchio y cols ${ }^{11}$, en un complejo protocolo, estudiaron en forma cruzada y randomizada a 53 enfermos que fueron programados ya sea a estimulación aurículo-BiV, estimulación aurículo-univentricular (VD o VI), y no estimulación, durante 4 semanas en cada modo. También estos autores encontraron que la estimulación con MP en modo aurículo-BiV se acompañó de mejoría de la CF, FE y calidad de vida durante los 12 meses de seguimiento. Cazeau y cols ${ }^{13}$ reportaron los resultados de 67 pacientes estudiados en forma randomizada y cruzada encontrando, adicionalmente, mejoría del peak de consumo de $\mathrm{O}_{2}$ y reducción de las hospitalizaciones por IC. Más recientemente Abraham et $\mathrm{al}^{25}$, reportaron los resultados del estudio MIRACLE, con los pacientes que completaron 6 meses de seguimiento, mostrando que la TRV se acompaña de mejoría de los índice de calidad de vida, CF incluyendo peak de consumo de $\mathrm{O}_{2} \mathrm{y}$, adicionalmente, disminución de las dimensiones del VI y del área de regurgitación mitral, comparado con el grupo control. Por lo tanto, y aunque el número de pacientes estudiados es aún limitado, la TRV 
parece útil en este subgrupo de pacientes con IC severa y asincronía de la contracción ventricular. En los enfermos tratados por nosotros, las variables clínicas analizadas muestran cambios porcentuales similares a los reportes recién comentados. Desgraciadamente, en nuestro hospital no disponemos de análisis más objetivos, como consumo de $\mathrm{O}_{2}$, lo que permitiría fundamentar más la mejoría, y eliminar, en parte, el eventual sefecto placebo", siempre presente en las distintas medidas terapéuticas.

En nuestra serie, la gran mayoría de los pacientes tratados estaban en ritmo sinusal, y existen muy pocos reportes de enfermos en FA. Aunque el beneficio hemodinámico de la sincronía $\mathrm{AV}$ junto a la optimización del IAV por Eco Doppler en pacientes con TRV es claro ${ }^{15}$, cuando hay FA sólo cabe esperar el beneficio que la corrección de la disincronía ventricular pueda tener. Nuestro único paciente en FA aumentó su FE de 25 a 38\% y su CF de III mejoró a II; otro enfermo que al someterlo a TRV estaba en ritmo sinusal, 2 semanas después cayó en FA y a pesar de perder la contracción auricular su FE se mantuvo en $25 \%$, y su capacidad de caminar en 6 min mejoró de 340 a 450 m y su CF actual es II. En ambos pacientes la contracción ventricular es completamente dependiente de la estimulación por los electrodos ventriculares pues no hay conducción a través del nódulo $\mathrm{AV}$, lo que ha sido definido como requisito para lograr una sbuena resincronización ${ }^{16}$. La mejoría clínica observada en estos casos es explicable por la corrección de la disincronía ventricular. Recientemente un grupo francés ${ }^{16}$, reportó una comparación de estimulación BiV en 22 enfermos en ritmo sinusal y 15 pacientes con FA, describiendo un mayor beneficio de la TRV en aquellos con FA. Por lo tanto, los pacientes con FA, que constituyen hasta $40 \%$ de los enfermos con $\mathrm{IC}^{27}$, también pueden ser favorecidos con esta terapia.

En relación a la etiología, los estudios indican que el beneficio en los enfermos coronarios es similar a los no isquémicos ${ }^{27}$. No obstante, es interesante que los 2 pacientes de nuestra serie que no tuvieron buena respuesta eran coronarios, con enfermedad residual de pequeño vaso, por lo que el factor isquemia en ellos puede ser importante.
Mortalidad en pacientes con TRV. Los pacientes con IC tienen una alta mortalidad cardiovascular y la causa de ésta se relaciona con la $\mathrm{CF}^{28}$; así, en CF I-II hay mayor mortalidad por MS arrítmica, en cambio en los enfermos con CF III - IV la principal causa de muerte es la IC. Si la TRV tendrá impacto en disminuir la mortalidad en estos casos está por definirse. Recientemente un metaanálisis realizado por Bradley y cols ${ }^{29}$ comunicó que esta forma de terapia mejora la sobrevida de pacientes con IC avanzada. De los estudios comentados con seguimiento más largo ${ }^{10-13}$, y de nuestra propia experiencia, cabe esperar que la sobrevida mejore. Los recientes hallazgos de evidencias de un remodelamiento ventricular reverso» con la TRV 22,24,30 orientan hacia el mismo sentido, y la extrapolación de otras medidas que inducen remodelamiento ventricular en pacientes con IC $^{31}$ también refuerzan esta hipótesis. Además, hay comunicaciones con menor incidencia de taquiarritmias ventriculares en pacientes con TRV ${ }^{32}$, lo que se atribuye a mayor estabilidad eléctrica por la mejor función ventricular.

La MS arrítmica continúa siendo un problema mayor en estos enfermos y aquellos que tienen riesgo arrítmico deben recibir un DI. Sin embargo, aparte de los enfermos con TV o fibrilación ventricular $(\mathrm{FV})$, no está definido qué pacientes con TRV debieran recibir un DI de resguardo ${ }^{33}$, lo que se espera sea resuelto por los estudios en marcha. En los 2 enfermos de nuestra serie que tuvieron MS no había antecedentes de arritmias ventriculares por lo que la TRV no incluyó DI, y ambos recibían amiodarona por antecedentes de taquiarritmias supraventriculares. Considerando la eficacia del DI en tratar la TV y FV es esperable que su adición disminuya la incidencia de MS arrítmica. Esto es particularmente importante si recordamos la alta incidencia de MS en pacientes con IC y que las arritmias ventriculares no sólo contribuyen a la alta mortalidad, sino que también favorecen la progresión de la $\mathrm{IC}^{31}$. En la reciente publicación del estudio ATLAS ${ }^{20}$, entre los pacientes que requirieron hospitalización por descompensación de IC, una proporción importante tuvo arritmia ventricular como factor descompensante. En los, hasta ahora pocos, enfermos con TRV y DI también se ha visto que las terapias antitaquicardia aplicadas a través de estimulación BiV son más 
eficaces que las aplicadas en $\mathrm{VD}^{34}$. Por lo tanto, la asociación de TRV con un DI podrían tener efectos sinérgicos entre sí, lo que debiera reflejarse en una mejor sobrevida.

Implicancias clínicas. La posibilidad de contar con una terapia que mejore la evolución de la IC severa es muy alentadora. Parece claro que en pacientes bien seleccionados hay mejoría aguda, en el corto y mediano plazo con TRV, pero aun no sabemos cuán duradera será ésta, y si cambiará la sobrevida. La posibilidad de una mejoría importante y sostenida por largo tiempo hace plantear que en algunos pacientes en lista de espera para trasplante pueda postergarse esa indicación, con las implicancias clínicas y económicas que esto tendría. En la actualidad, esto lo estamos investigando en un estudio latinoamericano (Protocolo ESPERA) sobre TRV en enfermos que están esperando un trasplante (estudio en desarrollo - datos no publicados).

Mención especial merece la utilidad de la TRV como «escate» en enfermos muy graves, ya sea postcirugía o como spuente» a un trasplante de urgencia, como ocurrió en 2 de nuestros pacientes. De hecho, uno de éstos actualmente está en CF II y se ha postergado la indicación de trasplante. Adicionalmente, y posterior al análisis del grupo de enfermos aquí presentados, otro paciente fue sometido a TRV de urgencia. Ese enfermo tenía un DI bicameral, llevaba 2 semanas en

\section{REFERENCIAS}

1. Braunwald E. Shattuck lecture: Cardiovascular medicine at the turn of the millenium: Triumphs, concerns and opportunities. N Engl J Med 1997; 337: 1360-9.

2. GoLdSTEIN S. Heart Failure Therapy at the turn of the Century. Heart Failure Rev 2001; 6: 27-41.

3. The antiarnythmics versus implantable defibrillators (AVID) Investigators. A comparison of antiarhythmic-drug therapy with implantable defibrillators in patients resuscited from near-fatal ventricular arrhythmias. N Engl J Med 1997; 337: 1576-83.

4. Buxton AE, LeE KL, Fisher JD, Josephson ME, Prystowsky EN, Hofley G. For the Multicenter Unsustained Tachycardia Trial (MUSTT) investigators. A randomized study of the prevention of espera de donante, con apoyo inotrópico máximo, a pesar de lo cual continuaba agravándose. Luego de implantar un electrodo en SC para TRV su condición mejoró y fue trasplantado un mes después. Esto es un tema no analizado en la literatura, probablemente porque en países desarrollados existe mayor disponibilidad de donantes y también sistemas de soporte circulatorio que permiten salvar estas emergencias.

Finalmente, debe definirse si en enfermos menos graves (CF II), pero que tengan disincronía y dilatación ventricular, la TRV puede modificar la evolución de la IC. De momento, se estima que esta terapia sería aplicable a aproximadamente $10 \%$ de todos los pacientes con IC, los que corresponden a aquellos con mayor compromiso funcional y trastorno de $\mathrm{CIV}^{26}$.

En conclusión: La presente serie de pacientes con IC severa refractaria y trastornos de la CIV o portadores de MP ventricular previo, muestra que la TRV mejora la función ventricular, lo que se asocia a mejoría de la CF y del test de caminata de $6 \mathrm{~min}$, efectos que se han mantenido en el seguimiento. La TRV ha sido bien tolerada y no se han observado efectos colaterales. La adición de DI podría evitar la MS arrítmica observada en 2 de nuestros enfermos. Se requiere mayor número de casos, seguimiento más prolongado y mayores estudios para evaluar si los efectos son más duraderos y si disminuye la mortalidad.

sudden death in patients with coronary artery disease. N Engl J Med 1999; 341: 1882-90.

5. Dressing TJ, Natale A. Congestive Heart Failure Treatment: The pacing approach. Heart Failure Rev 2001; 6: 15-25.

6. Shamim W, Francis DP, Yousufuddin M, Varney S, Pieopli MF, ANKer SD, Coats AJ. Intraventricular conduction delay: a prognostic marker in chronic heart failure. Int J Cardiol 1999; 70: 171-8.

7. Kerwin WF, Botvinick EH, O'ConNell JW, MerRick SH, DeMarco T, Chatterjee et al. Ventricular contraction abnormalities in dilated cardiomyopathy: effect of biventricular pacing to correct interventricular dyssynchrony. J Am Coll Cardiol 2000; 35: 1221-7.

8. Cazeau S, Ritter P, Lazarus A, Gras D, Backdach $\mathrm{H}$, Mundier O, Mugica J. Multisite pacing for end 
stage heart failure: Early experience. Pacing Clin Electrophysiol 1996; 19: 1748-57.

9. Lecierco C, Cazeau S, Le Breton H, Ritter P, Mabo P, GRAS D ET AL. Acute hemodynamic effects of biventricular DDD pacing in patients with end stage heart failure. J Am Coll Cardiol 1998; 32: 1825-31.

10. Gras D, Mabo P, Tang T, Luttikuis O, Chatoor R, Pedersen AK et aL. Multisite pacing as a supplemental treatment of congestive heart failure: preliminary results of the Medtronic Inc. InSync Study. Pacing Clin Electrophysiol 1998; 21: 224955.

11. Auricchio A, Stembrink C, Block M, Sack S, Vogt J, BAKKER P ET AL. Effect of pacing chamber and atrioventricular delay on acute systolic function of paced patient with congestive heart failure. The Pacing Therapies for Congestive Heart Failure Study Group. The Guidant Congestive Heart Failure Research Group. Circulation 1999; 99: 2993-3001.

12. Saxon LA, Boehmer JP, Hummel J, et Kacet S, DeMarco T, Naccarew G, Daoud E, For The Wigor CHF and Ventrak CHF InvESTIGator. Biventricular pacing in patients with congestive heart failure: Two prospective randomized trials. The VIGOR CHF and VENTAK CHF Investigators. Am J Cardiol 1999; 83: 120D-123D.

13. Cazeau S, Lecierco C, Lavergne T, Walker S, Varma C, LINDE C ET AL. Effects of multisite biventricular pacing in patients with heart failure and intraventricular conduction delay. N Engl J Med 2001; 344: 873-80.

14. Asenjo R, Oyarzún R, Morris R, Ortiz M, Morales $P$, Avalos V. Terapia de Resincronización Ventricular en Insuficiencia Cardíaca Avanzada (Abstract). Rev Chil Cardiología 2000; 19: 168.

15. Breithardt OA, Stembrink C, Franke A, Auricchio A, Houelle E, Sack S. On behalf of the pacing therapies for congestive Heart Failure Investigators. Echocandiographic evidence of hemodynamic and clinical improvement in patients pace for heart failure. Am J Cardiol 2000; 86 (Suppl): 133K-137K.

16. Lecierco C, Victor F, Alonso C, Pavin D, d'Allones $\mathrm{R}$, BANSARD JY ET AL Comparative effects of permanent biventricular pacing for refractory heart failure in patients with stable sinus rhythm or chronic atrial fibrillation. Am J Cardiol 2000; 85: 1154-6.
17. Herman MV, Heinle RA, Klein MD, Gordin R. Localized disorders in myocardial contraction. Asynergy and its role in congestive heart failure. N Engl J Med 1967; 277: 222-32.

18. Bramiet DA, Morris KG, Coleman RE, Albert D, Совв FR. Effect of rate-dependent left bundle branch block on global and regional left ventricular function. Circulation 1983; 67: 1059-65.

19. Grines CL, Bashore TM, Boudoulas H, Olson S, SHaFer P, Wooley CF. Functional abnormalities in isolated left bundle branch block. The effect of interventricular asynchrony. Circulation 1989; 79: 845-53.

20. XiAO HB, BRECKER SJ, GibSON DG. Effects of abnormal activation on the time course of the left ventricular pressure pulse in dilated cardiomyopathy. Br Heart J 1992; 68: 403-7.

21. Duncan AM, Wait TM, Gibson DG, Daubert C. The effect of biventricular pacing on ejection and filling hemodynamics in dilated cardiomyopathy patients with activation disurbances: The MUSTIC Study. J Am Coll Cardiol (Abstract) 2001; 37: 176A.

22. Yu Ch M, San ChP, Tse HF, Tang MO, Yang H, CHAU EMC ET AL. Reverse remodeling of the left ventricle after biventricular pacing in patients with advanced heart failure is related to intraventricular resynchronization: a mechanistic study by tissue Doppler imaging. J Am Coll Cardiol (Abstract) 2001; 37: 118A.

23. Leclerce C, Ronault G, Alonso C, Devilers A, DOUBERT C. Angioscintigraphic Assessment of cardiac resynchronization by biventricular pacing in heart failure patients. J Am Coll Cardiol (Abstract) 2001; 37: 393A.

24. Stellbrink C, Breithardt OA, Franke A, Sack S, BAKKer P, Auriechio A et al. Impact of cardiac resynchronization therapy using hemodinamically optimized pacing on left ventricular remodeling in patients with congestive heart failure and ventricular conduction disturbances. J Am Coll Cardiol 2001; 38: 1957-65.

25. Abraham WT, Fisher WG, Smith AL, Delurgio DB, LEÓN AR, LoH E et aL. Cardiac Resynchronization in Chronic Heart Failure. N Engl J Med 2002; 346: 1845-53.

26. Farwell D, Patel NR, Hall A, Ralph S, Sulke AN. How many people with heart failure are appropriate for biventricular resynchonization? Eur Heart J 2000; 21: 1246-50. 
27. Lecierco C, Alonso C, Pavin D, Ronault G, Mabo Ph, DAUBERT C. Comparative effects of ventricular resynchronization therapy in patients with LV systolic dysfunction of idiopathic or ischemic origin. (Abstract) J Am Coll Cardiol 2001; 37: 154A.

28. Carmona JR, BasterRa N. Prevención de muerte súbita en pacientes en espera de trasplante cardíaco. Rev Esp Cardiol 2000; 53: 736-45.

29. Bradiey DJ, Bradiey EA, Baughjahn KL, Berger RD, Calkins H, Goodman SN et al. Cardiac resynchronization and death from progressive heart failure. JAMA 2003; 289: 730-40.

30. Saxon LA, De Marco T, Schafer J, Chatterjee K, Kumar UNK, Foster E. Effects of long-term biventricular stimulation for resynchronization on echocardiographic measures of remodeling. Circulation 2002; 105: 1304-10.

31. Cieland JGF, Thygesen K, Uretsky BF, Armstrong P, HoRowitz JD, MasSIE B ET AL. Cardiovascular critical event pathways for the progression of heart failure. A report from the ATLAS study. Eur Heart J 2001; 22: 1601-12.
32. Higgins SL, Yong P, Scheck D, McDaniel M, Bownger F, VADECHA M ET aL. For the Ventak CHF Investigators. Biventricular pacing diminishes the need for ICD therapy. J Am Coll Cardiol 2000; 36: 824-7.

33. Gold MR, Peters RW. How great is the arrhythmic risk for heart failure patients and does resynchronization therapy modify it? En: Cardiac Arrhythmias 2001; Editado por Raviele A (Proccedings of the 7th International Workshop on Cardiac Arrhythmias- Venice, 7-10 october 2001) Springer, Milan, Italia. 151-6.

34. KüHLKAMP V. (For the Insync 7272 ICD) World Wide Investigators). Initial experience with an implantable cardioverter-defibrillator incorporating cardiac resynchronization therapy. J Am Coll Cardiol 2002; 39: 790-7.

\section{Agradecimientos}

Agradecemos a la Srta. Paola Muñoz C., por su colaboración en la preparación de este artículo. 